\title{
What do patterns in empirical data tell us about the structure of the world?
}

\author{
James W. McAllister
}

Received: 20 May 2009 / Accepted: 3 June 2009 / Published online: 7 July 2009

(C) The Author(s) 2009. This article is published with open access at Springerlink.com

\begin{abstract}
This article discusses the relation between features of empirical data and structures in the world. I defend the following claims. Any empirical data set exhibits all possible patterns, each with a certain noise term. The magnitude and other properties of this noise term are irrelevant to the evidential status of a pattern: all patterns exhibited in empirical data constitute evidence of structures in the world. Furthermore, distinct patterns constitute evidence of distinct structures in the world. It follows that the world must be regarded as containing all possible structures. The remainder of the article is devoted to elucidating the meaning and implications of the latter claim.
\end{abstract}

Keywords Empirical data $\cdot$ Evidence $\cdot$ Noise $\cdot$ Patterns $\cdot$ Phenomena $\cdot$ Structures

\section{Introduction}

Empirical science aims to ascertain the structure of the world. It does so by analyzing empirical data, consisting of the outcomes of observations and measurements, which constitute our sole epistemic access to reality. Empirical science must thus rest on what I will call a "principle of evidential correspondence" that relates structures in the world to features of empirical data. This principle licenses the move from perceiving a particular feature in a given empirical data set to claiming to have ascertained that the world has a particular structure.

The principle of evidential correspondence is central to the epistemology, methodology, and ontology of empirical science. Although we can interpret many debates among philosophers of science as addressing aspects of this principle, we have not yet seen a systematic attempt to give a general formulation of it. What can be said about

\footnotetext{
J. W. McAllister $(\varangle)$

Institute of Philosophy, University of Leiden, P.O. Box 9515, 2300 RA Leiden, The Netherlands e-mail: j.w.mcallister@let.leidenuniv.nl
} 
the content of the principle of evidential correspondence that underlies the modern empirical sciences?

One intuition on which we may draw is that empirical data in most cases must be analyzed into components before they reveal structures in the world. The global value of empirical data rarely delivers any clear evidence. This standpoint was first taken by Galileo Galilei, who thought that observed physical outcomes were the effect of the combination of one or more phenomena and of several perturbations: phenomena were fundamental constituents of reality that formed the object of his science and were to be described by laws of nature, whereas perturbations were irregular, ephemeral, and not amenable to systematic analysis. For example, Galileo regarded the fall of a body under everyday conditions as determined partly by a phenomenon, described by his law of free fall, and partly by various perturbations involving air resistance, the shape and composition of the body, and so on. Galileo concluded that observational data, since they recorded the resultant of all these causes, were often misleading: evidence about the fundamental structures of the world could be gathered only by analyzing data into components (Koertge 1977; McAllister 2005).

Present-day experimental scientists share Galileo's intuition. They assume that, while individual observations are affected by perturbations and errors, we can rely on the underlying constancies, regularities, and trends in empirical data to reveal structures in the world. The practice of repeating and varying experiments is designed partly to allow these features of data to shine through more clearly.

A great merit of the work of James Bogen and James Woodward (Bogen and Woodward 1988; Woodward 1989) is to have placed this intuition at the centre of discussions of scientific practice: they argue persuasively that the crucial feature of empirical data sets is the patterns that they exhibit, rather than the precise value of each data point. On Bogen's and Woodward's view, patterns in empirical data constitute evidence that the world contains particular fundamental and stable structures, which they dub "phenomena". Thus, detecting a particular pattern in an empirical data set licences the conclusion that the world contains a particular phenomenon. Correspondingly, the empirical content of laws and theories amounts to a description of certain potential patterns in data. This holds most clearly for so-called empirical laws, such as Kepler's laws and Snell's law, but the same applies to theories that are farther removed from the appearances. A law or theory is confirmed if, roughly, the relevant pattern is detected in data, and disconfirmed otherwise.

Building on Bogen's and Woodward's insight that patterns are the crucial evidential element of empirical data sets, this article aims to give a general formulation of the principle of evidential correspondence between features of empirical data and structures in the world. I will then suggest that the sole admissible principle of evidential correspondence leads to unexpected and radical conclusions about the structure of the world—conclusions that deviate markedly from those that Bogen and Woodward themselves draw.

\section{Patterns, noise, and their properties}

Empirical evidence comes in various forms. Many important bodies of data in present-day science take the form of strings of numbers, in which each number represents 
the outcome of a discrete measurement act. Other important data take the form of images, as Bogen (2009) notes. However, many images nowadays are digitally generated, and other images are used as a source of numerical data. Crystallographers, for example, extract from X-ray diffraction photographs numerical measurements of the angular positions and intensities of the individual spots, which constitute evidence for and against hypotheses about crystal structures. For these reasons, I will conceive empirical data in this article as strings of numbers.

If we conceive an empirical data set as a string of numbers, a pattern that is exhibited in a data set is an additive component of that string: it is what remains once some other component has been subtracted, item by item, from the data set (Grenander 1996).

Mathematically, there is no limit to the number and variety of patterns that are exhibited in a data set. This is because a string of numbers can be expressed as the sum of any string whatever and a second string that matches the discrepancy between the two. Drawing on the metaphor of signalling theory, I shall call the discrepancy between a data set and a pattern identified within it the "noise" with which that pattern is exhibited in that data set. The term "noise" thus denotes purely the mathematical difference between the original data set and the pattern into which one has chosen to decompose the data set.

Given that, mathematically, an empirical data set can be decomposed into any one of all conceivable patterns and an associated noise term, what does this fact reveal about the structure of the world? Providing a full answer to this question goes a long way towards specifying the content of the principle of evidential correspondence defined in the previous section. There would seem to be two possible initial responses. One is to claim that only some of the patterns that we can discern in data sets correspond to structures in the world, and then to specify the respect in which patterns that correspond to structures in the world differ from patterns that do not. The other response is to deny that any such ontologically significant distinction between patterns can be drawn, to admit that all patterns exhibited in empirical data sets correspond to structures in the world, and then to consider the meaning and implications of this claim.

Let us begin by exploring the first option. Most writers in philosophy of science assume that only some of the patterns exhibited by empirical data sets correspond to structures in the world, whereas other patterns that could be discerned in empirical data have no evidential significance. Bogen and Woodward are among them. They hold that the world contains only a relatively small number of the structures that they call phenomena, and they do not explicitly raise the possibility that the world contains further structures of other kinds. In the light of this, they believe that only a few of the patterns that might be discerned in empirical data sets are evidence of structures in the world. Indeed, their examples suggest that they think that no more than one pattern in a typical empirical data set corresponds to a phenomenon.

In order to avoid circularity, however, those who take this option must specify the respect in which patterns that correspond to structures differ from patterns that do not. If Bogen and Woodward cannot specify which patterns correspond to what they call phenomena, they have no justification for believing that the world contains a certain set of phenomena rather than a different set (McAllister 1997). For example, in order to substantiate his claim that it is an objective fact that some but not all patterns in data correspond to "true causes", Bogen (2009) must explain how they differ. 
In what respect does a pattern exhibited by an empirical data set that corresponds to a structure in the world differ from a pattern that does not? In attempting to answer this question, we operate under a restriction: we cannot appeal to knowledge about which structures the world contains. If we could, we would be able to say simply that the patterns in empirical data that have evidential significance are those that correspond to the list of verified structures in the world. In fact, this answer presupposes what we are attempting ultimately to ascertain — what structures the world contains. Instead, in answering this question, we are restricted to using information contained in empirical data themselves, since empirical data constitute our sole epistemic access to the world and its structures. We must induce criteria for distinguishing patterns that correspond to structures in the world from patterns that do not in some way from the empirical data themselves. From this, it follows that we must be able to specify the difference between patterns that correspond to structures in the world and patterns that have no evidential significance purely in terms of morphological parameters of the patterns themselves or of the noise terms with which they are exhibited in data.

The infinitely many patterns into which one can decompose a given empirical data set can be ranked and assessed on various morphological parameters. One is the algorithmic complexity of the pattern. A pattern of high algorithmic complexity admits no description substantially shorter than the data set itself, whereas a pattern of low algorithmic complexity is highly regular and thus can be captured in a much shorter description ( $\mathrm{Li}$ and Vitányi 1997). A second parameter is the magnitude of the noise term, or of the discrepancy between the pattern and the original data set. A pattern exhibited with lower noise will account for a larger part of the variance present in the data set, whereas a pattern exhibited with higher noise will account for a smaller part of that variance. A third parameter is the degree to which the noise term associated with the pattern shows patterns of its own, including correlations with the pattern picked out from the data. For example, if the values in a time series have roughly constant magnitude, and we pick out from that data set a pattern described by a sine curve, the associated noise term will show a pattern corresponding to the inverse of that sine curve.

We might hope that the difference between patterns that correspond to structures in the world and patterns that have no evidential significance would manifest itself in different values of one or more of these morphological parameters. A natural suggestion is to say that the patterns that correspond to structures in the world differ from patterns that do not in respect of the noise terms with which they are exhibited in empirical data: for example, that a pattern fails to correspond to a structure if the noise with which it is exhibited is high, shows patterns of its own, or is not randomly distributed. Woodward (2009), for example, suggests that a pattern fails to correspond to what Bogen and he call a phenomenon if it is exhibited with noise that is not randomly distributed.

These proposals seem initially to accord with a simplified model of inferential statistics, but they are undermined partly by systematic considerations and, more crucially, by examples of scientific practice. Before we turn to the examples, let us review two systematic considerations that limit the scope of these proposals.

First, it is not sufficient to stipulate conventional numerical thresholds of these parameters, saying, for example, that a pattern corresponds to a structure in the world 
only if it is exhibited in an empirical data set with noise no higher than $10 \%$. Such a conventional stipulation might be adequate if we required a pragmatic, methodological guide to data handling; but we presume that the partition between patterns that correspond to structures in the world and patterns that have no evidential significance depends solely on the structure of the world, and we should not expect a conventional stipulation to capture it.

Second, it is not adequate to say that, given two patterns exhibited in a data set, only the pattern that is exhibited with lower noise corresponds to a structure in the world. It is easy to pick out a pattern that is exhibited by a data set with zero noise: this is the unique pattern of high algorithmic complexity that coincides precisely with the original data. We would not want to conclude that this pattern alone corresponds to a structure in the world, especially in the light of the fruitful intuition that the value of individual data points is less significant than the underlying constancies, regularities, and trends in empirical data (McAllister 2003).

Let us now turn to examples of scientific practice. Scientists are ready to attribute evidential significance to patterns in data that have low and high algorithmic complexity, to patterns that are exhibited with low and high noise, and to patterns that are exhibited with noise that shows patterns of its own, including correlations with the pattern picked out from the data. They are willing to regard patterns with all these characteristics as corresponding to real structures in the world (McAllister 2007).

Let us consider a data set consisting of microwave radiation intensity readings of the sky. Cosmologists have identified multiple different patterns in this data set, each of which is exhibited with a different noise level; furthermore, they regard each of these patterns as evidence of a real structure in the universe. One pattern, named "isotropic background", consists of a constant intensity distribution, independent of direction, with the spectrum of a black body at approximately $2.7 \mathrm{~K}$. Cosmologists regard this pattern as evidence of the decoupling of matter and radiation in the early universe. A second pattern, named "dipole anisotropy", consists of one warm and one cool pole in opposite directions in the sky, and is modelled by a first-order spherical harmonic function with an amplitude of approximately $3.4 \times 10^{-3} \mathrm{~K}$. Cosmologists regard this pattern as evidence of the motion of the earth relative to the matter that last scattered the microwave radiation. A third pattern, dubbed "quadrupole", consists of two warm and two cool poles, and is modelled by a second-order spherical harmonic function with an amplitude of around $1.6 \times 10^{-5} \mathrm{~K}$. Cosmologists attribute this pattern to the radio emissions of the Milky Way. A fourth pattern, called "ripples", consists of irregular fluctuations of radiation temperature with an amplitude of approximately $3.1 \times 10^{-5} \mathrm{~K}$ on angular scales of $10^{\circ}$ and above. Cosmologists regard this pattern as evidence of inhomogeneities in the matter distribution in the early universe, from which galaxies and clusters of galaxies condensed. The fifth pattern identified up to now, dubbed "acoustic oscillations", consists of irregular fluctuations of radiation temperature with an amplitude of approximately $6.9 \times 10^{-5} \mathrm{~K}$ on an angular scale of around $1^{\circ}$. Cosmologists regard this pattern as evidence that the universe is flat, i.e. that the total mass and energy density of the universe is equal to the so-called critical density (Partridge 1995; Weinberg 2008).

The patterns in this series are exhibited with noise terms that show all the properties that might conceivably be regarded as indicating that a pattern has no evidential 
significance. First, consider the suggestion that a pattern fails to correspond to a structure in the world if it is exhibited in a data set with high noise. The pattern named "isotropic background" is exhibited in the cosmic microwave radiation data with relatively high noise- much higher than the noise with which any of the other patterns is exhibited. This does not weaken the belief of cosmologists that this pattern is evidence of the decoupling of matter and radiation in the early universe. Next, consider the suggestion that a pattern has no evidential significance if it is exhibited in a data set with noise that shows patterns of its own. Since the five patterns listed above are all superposed on one another in the cosmic microwave radiation data, it follows that, after we have subtracted any one of these patterns from the data, the remainder-which counts as the noise term with which the subtracted pattern is exhibited in the datashows various further patterns. This does not stop cosmologists taking each of these patterns separately as evidence of a distinct physical process in the universe. Lastly, consider Woodward's suggestion that a pattern fails to correspond to a phenomenon if it is exhibited in a data set with noise that is not randomly distributed. The pattern named "acoustic oscillations" is exhibited in the cosmic microwave radiation data with a noise term that, among other things, shows the pattern named "dipole anisotropy", and therefore cannot be described as randomly distributed. This does not dissuade cosmologists from regarding the pattern named "acoustic oscillations" as evidence of the flatness of the universe.

The mining of cosmic microwave radiation data for evidence about cosmological processes undoubtedly counts as one of the paradigmatic examples of good science in the twentieth century. If we were to discard patterns that are exhibited in data sets with noise terms that are high, show patterns of their own, or are not randomly distributed, we would disqualify this achievement. This observation suggests that the above account of the difference between patterns that correspond to structures in the world and patterns that do not cannot be correct: patterns that fail the test have no less claim to evidential significance than patterns that pass it.

One could claim that the cosmic microwave radiation data are unusually rich, and that other empirical data sets are simpler or more univocal. In fact, the opposite is the case. It is usual for empirical data sets to exhibit multiple patterns with different noise levels, each of which is regarded by scientists as evidence of a distinct structure in the world. Take, as a second example, the data set comprising all atmospheric temperature measurements made on earth. This exhibits a pattern with a period of a day, associated with the earth's rotation about its axis; a pattern with a period of a year, associated with the earth's orbit around the sun; several patterns with periods ranging from 11 to 100,000 years, attributed to various astronomical processes; several less regular patterns associated with individual weather systems, which have a life span of a few days or weeks; patterns associated with El Niño events, occurring at intervals of between 4 and 10 years; and the "hockey stick" pattern associated with anthropogenic changes in the composition of the atmosphere in the twentieth century (Mann et al. 1998; Burroughs 2003; Ruddiman 2008). This series of patterns reproduces all the features shown by the cosmic microwave radiation data. The "hockey stick" pattern, for example, is exhibited with much higher noise than the pattern representing daily temperature excursions; moreover, this noise term shows patterns of its own and is not randomly distributed, since it contains all the other patterns listed above. If we accepted 
the above account of the difference between patterns that correspond to structures in the world and patterns that do not, we would have to conclude that the "hockey stick" pattern is not evidence of anything in the world - a conclusion that few nowadays would embrace.

Perhaps alternative accounts of the difference between patterns that correspond to structures in the world and patterns that do not can be proposed. We would have to scrutinize any such new proposal with care. The list of paradigmatic examples of good science is sufficiently heterogeneous, however, that we are likely to find examples counting against any such proposal.

\section{Objections from the temporal development of science}

Some critics might argue that so far I have taken insufficient account of the development of science in time. Perhaps later discoveries build on earlier discoveries to reveal which patterns in data correspond to structures in the world and which lack evidential significance. There are two ways in which the temporal development of science might enable scientists to distinguish patterns that correspond to structures in the world from patterns that do not.

First, as Woodward (2009) points out, scientists approach empirical data normally not as blank slates, but equipped with substantive assumptions about the world. Woodward suggests that, since these assumptions go beyond the empirical data under scrutiny, they allow us to infer which patterns exhibited in those data constitute evidence of phenomena. In many cases, these assumptions entail that one should seek patterns of a certain form. Consider, for example, a data set listing the temperatures at which samples of lead have been observed to melt in a series of trials. If we assume that the melting point of a chemical element depends only on the atomic number of the element, we might infer that the sole pattern in a graphical plot of this data set that constitutes evidence about the structure of the world is a horizontal straight line.

Assumptions of this kind, while they play an important pragmatic role in scientific research, are unable to resolve the ontological question of which structures the world contains. This would certainly hold if these assumptions were stipulations: stipulations cannot be expected to latch onto a putative objective feature of the world, namely the structures that the world contains. Woodward, of course, considers these assumptions to be not stipulations but hypotheses that command empirical support. Such assumptions do not help either, however. The empirical support for an assumption can take the form only of a pattern identified in an empirical data set on a previous occasion. For example, the empirical support for the assumption that the melting point of a chemical element depends only on the atomic number of the element takes the form of a pattern consisting of another horizontal straight line exhibited in a graphical plot of empirical data on the melting of samples of various elements. The questions that I raised above about the later data set apply to this earlier data set too: what is the difference between patterns that correspond to structures in the world and patterns that do not? Reference to empirically supported assumptions about the structure of the world, therefore, does no more than advance the problem to an earlier time. 
The second feature of the temporal development of science that might enable scientists to distinguish patterns that correspond to structures in the world from patterns that do not is the discovery that some patterns are projectable, whereas others are not. The argument might go as follows: it is easy for a scientist to fit multiple patterns to data that are already available, but the real test is faced when he or she checks these patterns against data gathered in future. Then, the scientist will discover that some previously identified patterns are exhibited also in the new data, whereas other patterns are not. It seems natural to suggest that the former patterns have greater claim to correspond to structures in the world than the latter.

What does the discovery that a pattern is or is not projectable amount to? The statement that a pattern $P(x)$ is projectable means that, if $P(x)$ is exhibited with a noise level of $n$ percent in a data set $A$, it will later be found to be exhibited with a noise level no higher than $n$ percent also in a second data set $B$. By contrast, the statement that a pattern $Q(x)$ is not projectable means that, whereas $Q(x)$ is exhibited with a noise level of $m$ percent in $A$, it will later be found not to be exhibited with a noise level no higher than $m$ percent in $B$.

These outcomes may represent an interesting discovery about the two patterns, but they provide no reason for thinking that pattern $P(x)$ has more claim to correspond to a structure in the world than pattern $Q(x)$. From findings about patterns in data sets established above, it is clear that the data set $B$ exhibits both $P(x)$ with a certain noise level and $Q(x)$ with a certain other noise level. Insofar as patterns in data are regarded as corresponding to structures in the world, these two patterns have equal claim. The fact that at some moment we predicted that $P(x)$ would be exhibited in $B$ with a noise level no higher than $n$ percent, and this prediction was later verified, and we predicted that $Q(x)$ would be exhibited in $B$ with a noise level no higher than $m$ percent, and this prediction was later not verified, has no bearing on the ontological question which patterns in data correspond to structures in the world and which do not. It merely causes us to revise our estimate of the noise level with which $Q(x)$ is exhibited. For projectability to offer a criterion to distinguish patterns that correspond to structures in the world from patterns that do not, it would have to be supplemented with an argument for thinking that all and only patterns exhibited with a noise level no higher that $n$ percent correspond to structures in the world. The projectability proposal, in short, far from offering a solution to the problem before us, presupposes such a solution.

The irrelevance of the temporal succession of prediction and verification to the ontological question may be established also in another way. Consider the data set $U$ comprising all observations that we will in fact make throughout the history of science, or even comprising all observations that we could make in the actual world. Presumably $U$ provides the most reliable empirical base possible for our project of establishing from empirical evidence which structures the world contains. Now, all the results about patterns exhibited in data sets established above hold of the patterns exhibited in $U$. In particular, $U$ exhibits all possible patterns, each with a certain noise level. Some writers, like Bogen and Woodward, would claim that some of the patterns exhibited in $U$ correspond to structures in the world, and other patterns do not. These writers face the following question: what is the difference between patterns of these two classes? It is evident that historical circumstances pertaining to the gradual process 
by which we ascertain the noise levels with which individual patterns are exhibited in $U$ cannot provide grounds for resolving this question.

\section{The equality of patterns}

Let us now turn to the other possible response to the fact that, mathematically, an empirical data set can be decomposed into any one of all conceivable patterns and an associated noise term. This second response denies that any ontologically significant distinction can be drawn between patterns, and concludes that all patterns exhibited in empirical data sets constitute evidence of structures in the world. What grounds are there for endorsing this response?

The grounds for thinking that all patterns that can be discerned in an empirical data set correspond to structures in the world are the following. A pattern is an additive component of an empirical data set. If the world causes the full value of the empirical data, as it must, then some factor in the world causes every additive component of that value. Whereas it may be difficult, from a pattern in data, to reconstruct the particular element of the combination of causal factors in the world that produced it, it is incontrovertible that such a factor exists and that the pattern is an effect of it. For example, each of the patterns that cosmologists have identified in cosmic microwave radiation data - the isotropic background, the dipole anisotropy, the quadrupole, the ripples, and the acoustic oscillations-is caused by a particular physical process. A pattern in data is a trace of the causal factor that produced it. Therefore, every pattern exhibited in empirical data constitutes evidence of some structure in the world.

One might object that, unlike in the case of the patterns just mentioned, we have not yet theorized distinct causal factors that are responsible for the vast majority of patterns that we might identify in empirical data. This, however, can be explained as reflecting the incompleteness of current science. A further possible objection, that there is no evidence of the existence of a distinct physical factor responsible for an arbitrary pattern that we may discern in empirical data, is misconceived: the pattern itself constitutes such evidence. For example, the evidence of the existence of physical processes responsible for the patterns identified in cosmic microwave radiation data takes the form of the patterns themselves.

The conclusion that every pattern exhibited in empirical data constitutes evidence of some structure in the world holds regardless of the algorithmic complexity of the pattern, the magnitude of the noise with which it is exhibited in the data set, and whether this noise shows patterns of its own. In particular, nothing about the noise with which any pattern is displayed in a data set lowers its worth as evidence of structure in the world: even a pattern that is displayed with high noise is the effect of a causal process in the world, and thus constitutes evidence of that process.

We may test this view further by scrutinizing one of its implications. According to this view, there is no component of any empirical data set that does not constitute evidence about the structure of the world. If this view is correct, it follows that even what is labelled, for the purposes of a certain research project, as the noise term in a data set contains information about some structure in the world, albeit not one that lies at the focus of that project. In other words, for any component of an empirical data set that counts for one research project as noise, it must be possible to formulate another 
project for which that component constitutes the pattern of interest and the source of evidence. Is this implication confirmed by our experience of scientific research?

I believe it is. There are countless examples of the discovery of useful information in what in other contexts is classed as noise in data. For Arno A. Penzias and Robert W. Wilson, who set out to investigate the reflection of radio waves by satellites, the sum of all the patterns now found in cosmic microwave radiation data counted originally as noise. Small fluctuations in observational data in some research projects are attributed to Brownian motion and are discarded as noise, but a research project into the properties of matter will regard them as evidence of the existence and properties of atoms, from which Avogadro's number can be calculated. Voltage fluctuations in an electrical resistor are discarded in many research projects as thermal noise or Johnson noise, but may be used in another research project as an indicator of temperature (White et al. 1996).

Indeed, scientific progress is often achieved by re-analyzing the component of empirical data that was discarded as noise in earlier research and showing that it contains information about further structures of the world. As we saw above, Galileo analyzed observational data on the fall of bodies into two components: one that was described by his law of free fall, and a second that he regarded as too irregular to yield useful information. In the nineteenth century, we discovered that this second component contained important further patterns, described by Stokes's law. Such examples suggest that the partition of a data set into pattern and noise is context dependent and does not correspond to any objective difference between causal factors in the world (Kosko 2006, pp. 6-8).

This test confirms the thesis that there is no element of empirical data sets that does not constitute evidence about the structure of the world. This enables us to give a preliminary formulation of the principle of evidential correspondence relating features of empirical data and structures in the world on which empirical science necessarily relies: all patterns exhibited in empirical data, irrespective of the magnitude or other features of the noise with which they are exhibited, correspond to structures in the world.

\section{From patterns to structures}

Let us suppose that the preliminary formulation of the principle of evidential correspondence just given is correct, and that all the endlessly many patterns into which a data set can be decomposed constitute evidence of the structure of the world. What substantive conclusions about the structure of the world does this entail?

Any pattern into which we can decompose a data set can be expressed as the constancy of a certain mathematical function. That is, all patterns in data can be expressed in the form $F_{i}(x)=0$. Consider now a particular pattern, $F_{1}(x)=0$. What evidence about the structure of the world does this pattern contain? This pattern is evidence that the world has the invariance or symmetry necessary to cause data to exhibit this particular constancy. In other words, there must be some causal process in the world that ensures that the quantity $F_{1}(x)$ remains equal to zero.

The principle of conservation of energy offers a good illustration. We discover that the world has the structure called "conservation of energy" by detecting that certain empirical data sets exhibit, with non-zero noise, a pattern $E(x)=0$. From this pattern, 
we infer that the world contains a combination of causal processes that ensures that the quantity $E(x)$ remains constant. Physicists describe these, of course, as the causal processes by which energy is converted from one form into another within closed physical systems. Thus, the pattern $E(x)=0$, exhibited in empirical data sets with non-zero noise, constitutes evidence that the world has a certain invariance. The same holds for all other patterns of the form $F_{i}(x)=0$.

Thus, from the fact that empirical data show all possible patterns, we must conclude that the world contains invariances that cause all possible patterns. How many distinct invariances are these? Are these all possible invariances, or merely a finite number? In other words, is there a one-to-one relation between invariances in the world and patterns in data, or a one-to-many relation?

An answer to this question will be provided by a criterion for individuating invariances in the world. If our individuation criterion counts the invariances that cause two distinct patterns in data as distinct, a one-to-one relation between invariances and patterns will hold; if our criterion in some cases counts those invariances as the same, a one-to-many relation will hold.

Now, our sole possible epistemic access to invariances is through the patterns in empirical data that they cause. I therefore suggest that we make our individuation criterion for invariances follow our individuation criterion for patterns in data: if two patterns differ, then the invariances that caused them differ also. On this criterion, there are as many distinct invariances in the world as there are distinct patterns in empirical data.

The alternative view, that, in some cases, multiple distinct patterns are caused by the same invariance, is open to three objections. First, this view is empirically unmotivated. This view envisages a taxonomy in which patterns are attributed to a smaller number of invariances. However, there is no empirically detectable fact that can weigh for or against any such taxonomy. Second, this view does not amount to a coherent alternative. An invariance that manifests itself in multiple distinct patterns in data must be regarded as complex: it has multiple facets, each of which causes one of the patterns. If we are willing to go this far, we may equally identify each of its facets with an invariance in its own right. Third, this view is not stable. If we assume that an invariance is able to cause multiple patterns in data, then simplicity considerations will favour saying that the world in its entirety consists of just one invariance, which causes all the patterns in data that we are capable of picking out. Then, the option of saying that this invariance has as many facets as the patterns that we discern in empirical data becomes all the more compelling.

These critical considerations support the conclusion that distinct patterns in empirical data constitute evidence of, and in this sense correspond to, distinct invariances in the world. Given that the class of invariances is equivalent to the class of structures, it follows that empirical data constitute evidence for the proposition that the world contains all possible structures.

Let us briefly recapitulate the argument up to now:

- Empirical data sets exhibit all possible patterns, each with a certain noise term.

- Each pattern in empirical data, irrespective of its properties and of those of the noise term with which it is exhibited in data, is evidence of a structure in the world. 
- Distinct patterns in data are evidence of distinct structures in the world.

- Therefore, empirical data constitute evidence that the world contains all possible structures.

We can now add detail to the preliminary formulation of the principle of evidential correspondence given at the end of Sect. 4. Our formulation then was that all patterns exhibited in empirical data correspond to structures in the world. We can now state the principle of evidential correspondence more fully as follows:

Principle of evidential correspondence: Distinct patterns exhibited in empirical data, irrespective of the magnitude or other features of the noise with which they are exhibited, correspond to distinct structures in the world.

Coupled with the finding that empirical data contain all possible distinct patterns, this principle yields the conclusion that the world contains all possible structures.

\section{A radically polymorphous world}

I propose to label a world that contains all possible structures as "radically polymorphous". The thesis that the world is radically polymorphous is alien to most thinking in philosophy of science and to our everyday conceptions of the world. Nonetheless, I believe that it is the sole possible outcome of a careful analysis of the features of empirical data and their evidential significance. In this section, I attempt to make the thesis more palatable by neutralizing some possible misgivings at it.

How should we conceptualize the simultaneous existence of all structures in the world? Since our sole possible evidence that the world has some structure comes in the form of patterns in empirical data sets, I suggest that we allow our understanding of structures in the world to be guided by our understanding of patterns in data. The fact that we pick out one pattern in a data set does not establish that alternative patterns do not exist. In actuality, as we have seen, a data set exhibits all possible patterns with various noise levels. Patterns are superposed on one another and exist simultaneously in data: they are all present in the same empirical data set, available to be picked out or to be disregarded. We ought to conceive of structures in the world in the same way. Whereas we habitually think of a structure as something incompatible with alternative structures, in actuality all structures exist simultaneously in the world, available to be picked out or to be disregarded.

Some might label this a form of relativism, but this characterization is wide of the mark. Relativism would involve saying that which structure the world has, depends on the observer's standpoint. By contrast, I hold that the world has all structures absolutely: all structures are objectively real for all observers. Whatever its inadequacies, therefore, this is not a form of relativism. At most, only Bogen's and Woodward's label "phenomenon"- now reduced to an honorific title designating the structure that is at the focus of a particular research project-shifts from structure to structure depending on the research project being pursued, just as what counts as the pattern and as the noise in data depends on the project.

A possible extension of this view is to reason that an entity that exhibits all possible symmetries is equivalent to one that has no structure at all, and conclude that the world 
is amorphous. Whereas I would not quarrel with this further conclusion, I do not at present see any advantages in adding this.

In virtually all practical cases, of course, observers choose to pick out just one pattern at a time from the infinitely many that are available in empirical data. Two devices in the observer normally guide the choice. The first is a preference for patterns of a certain form. For example, an observer may choose to pick out a pattern consisting of a horizontal straight line, corresponding to the constancy of some physical magnitude, from a graphical plot of a data set. As we saw in Sect.3, the choice to pick out a pattern of a certain form in a data set may be motivated or constrained by previous choices to pick out certain other patterns in other data sets. In this sense the choice of a pattern of a particular form may be empirically motivated, although no history of earlier choices of patterns establishes that the latest pattern chosen has any more claim than any other pattern to correspond to a structure in the world.

The second device is a preference for a noise level. An observer may tune in to patterns that are exhibited in a data set with higher or lower noise. A preference for a noise level enables the observer to choose whether to focus on patterns that follow the variance of the data more or less precisely. In many cases we would describe a pattern that is exhibited with lower noise as corresponding to smaller-scale structures in the world, and patterns exhibited with higher noise as corresponding to larger-scale structures.

Preference for a noise level is built in to the human senses and to all observational instruments. For example, the human sense of sight has a preference for a noise level that ensures that we interpret visual stimuli in terms of structures of a size of approximately one meter. If the preferred noise level were lower, we would focus on smaller-scale structures, becoming more aware of the irregularities of a tabletop, for example. If the preferred noise level were higher, we would lose sight of many smaller objects altogether, and see landscapes as flat and bare. Structures on these alternative scales-irregularities of tabletops, featurelessness of landscapes-are self-evidently present too, but human observers in everyday circumstances usually focus on them less than on mid-sized structures.

An observer's preference for patterns of a certain form or for a certain noise level leads that observer to focus on some patterns in data and thus on some structures in the world to the exclusion of others. Neither preference, however, has any bearing on the question whether the world contains just one structure or, as I maintain, all possible structures. This question is decided exclusively by two factors: which patterns are exhibited in empirical data, and what evidential significance these patterns have. Indeed, observers would not need preferences for patterns of a certain form and for a certain noise level if the world manifestly displayed a unique structure.

On a psychological level, the facility to pick out a single pattern may give observers the impression that the world has a unique structure, but this impression is corrected if they realize that they might equally legitimately focus on another pattern in the same empirical data. Whereas some researchers might regard science as the project to identify the unique structure of the world, anyone with experience of complex data sets - such as those pertaining to the cosmic microwave radiation or atmospheric temperature - will acknowledge that empirical data themselves contradict the assumption that the world shows a unique structure. A world with a unique 
structure would not generate empirical data with the properties that we find data to have.

Lastly, the options for those who wish to block the conclusion that the world has all possible structures are clear. They must either establish that empirical data sets do not exhibit all possible patterns, or propose a formulation of the principle of evidential correspondence between features of empirical data and structures in the world alternative to that given above. To do the latter, they must establish either that some of the patterns exhibited by empirical data sets do not constitute evidence of structures in the world, or that multiple distinct patterns constitute evidence of the same structure in the world. As I have tried to argue in the preceding pages, I believe that none of these challenges can be met.

\section{Conclusions}

In this article, I have directed attention at the need to formulate the principle of evidential correspondence underlying modern empirical science. This principle is required to licence the move from perceiving a certain feature in an empirical data set to concluding that the world contains a certain structure. The principle should specify which features of empirical data constitute evidence of which structures in the world.

I have argued that the sole admissible principle of evidential correspondence states that distinct patterns exhibited in empirical data correspond to distinct structures in the world. This principle holds irrespective of the magnitude or other properties of the noise with which patterns are exhibited in data sets.

I have coupled this principle with the finding that empirical data sets exhibit all possible patterns, each with a certain noise level. My conclusion is that the world contains all possible structures. I have dubbed such a world "radically polymorphous".

Whereas the thesis that the world is radically polymorphous appears at first sight to conflict with our everyday experiences and with the findings of science, I have argued that this thesis is actually required to make sense of the nature of empirical data, and especially of its inexhaustibility. Whereas more work is needed to explore the implications of this view, it seems to suggest a new conceptualization of the way multiple structures co-exist in the world.

Acknowledgements I dedicate this article to the memory of Daniela Bailer-Jones (1969-2006), who established and led the research programme, "Kausalität, Kognition und die Konstitution naturwissenschaftlicher Phänomene", at the Ruprecht-Karls-Universität Heidelberg. I presented a first draft at the conference, "Data-Phenomena-Theories: What's the Notion of a Scientific Phenomenon Good for?", Heidelberg, September 2008. I thank the organizers for inviting me to speak and the participants for valuable comments. I am especially grateful to James Bogen and James Woodward for cordial and constructive exchanges.

Open Access This article is distributed under the terms of the Creative Commons Attribution Noncommercial License which permits any noncommercial use, distribution, and reproduction in any medium, provided the original author(s) and source are credited.

\section{References}

Bogen, J. (2009). 'Saving the phenomena' and saving the phenomena. Synthese. doi:10.1007/ s11229-009-9619-4. 
Bogen, J., \& Woodward, J. (1988). Saving the phenomena. Philosophical Review, 97, 303-352.

Burroughs, W. J. (2003). Weather cycles: Real or imaginary? (2nd ed.). Cambridge: Cambridge University Press.

Grenander, U. (1996). Elements of pattern theory. Baltimore, MD: Johns Hopkins University Press.

Koertge, N. (1977). Galileo and the problem of accidents. Journal of the History of Ideas, 38, 389-408.

Kosko, B. (2006). Noise. New York: Viking.

Li, M., \& Vitányi, P. M. B. (1997). An introduction to Kolmogorov complexity and its applications (2nd ed.). Berlin: Springer.

Mann, M. E., Bradley, R. S., \& Hughes, M. K. (1998). Global-scale temperature patterns and climate forcing over the past six centuries. Nature, 392, 779-787.

McAllister, J. W. (1997). Phenomena and patterns in data sets. Erkenntnis, 47, 217-228.

McAllister, J. W. (2003). Algorithmic randomness in empirical data. Studies in History and Philosophy of Science, 34, 633-646.

McAllister, J. W. (2005). The virtual laboratory: Thought experiments in seventeenth-century mechanics. In H. Schramm, L. Schwarte, \& J. Lazardzig (Eds.), Collection, laboratory, theater: Scenes of knowledge in the 17th century (pp. 35-56). New York: De Gruyter.

McAllister, J. W. (2007). Model selection and the multiplicity of patterns in empirical data. Philosophy of Science, 74, 884-894.

Partridge, R. B. (1995). 3 K: The cosmic microwave background radiation. Cambridge: Cambridge University Press.

Ruddiman, W. F. (2008). Earth's climate: Past and future (2nd ed.). New York: W. H. Freeman.

Weinberg, S. (2008). Cosmology. Oxford: Oxford University Press.

White, D. R., Galleano, R., Actis, A., Brixy, H., De Groot, M., Dubbeldam, J., et al. (1996). The status of Johnson noise thermometry. Metrologia, 33, 325-335.

Woodward, J. (1989). Data and phenomena. Synthese, 79, 393-472.

Woodward, J. (2009). Data and phenomena: A restatement and defense. Synthese. doi:10.1007/ s11229-009-9618-5. 